\title{
Phenomenological viability of string inspired multi-Higgs doublet models
}

\author{
Ana Teixeira* ${ }^{* \dagger}$ \\ Departamento de Física Teórica C-XI and Instituto de Física Teórica C-XVI, \\ Universidad Autónoma de Madrid, \\ Cantoblanco, E-28049 Madrid, Spain \\ E-mail: teixeira@delta.ft.uam.es
}

\begin{abstract}
We analyse the phenomenological viability of heterotic $Z_{3}$ orbifold models with two Wilson lines, which naturally predict three families of matter and Higgs fields. We study the orbifold parameter space, and discuss the compatibility of the predicted Yukawa couplings with current experimental data, thus evaluating the viability of the orbifold configurations. We address the implications of tree-level flavour changing neutral processes in constraining the Higgs sector of the model, finding that viable scenarios can be obtained for a fairly light Higgs spectrum.
\end{abstract}

International Europhysics Conference on High Energy Physics

July 21 st - 27th 2005

Lisboa, Portugal

\footnotetext{
* Speaker.

$\dagger$ Based on work done in collaboration with C. Muñoz and N. Escudero. Work supported by "Fundação para a Ciência e Tecnologia”, under grant SFRH/BPD/11509/2002.
} 


\section{Introduction}

Even though the standard model (SM) has proved to offer a very successful description of strong and electroweak interactions, it fails in providing an explanation to issues such as the gauge group, the number of families, the dynamics of flavour and the mechanism of mass generation, among others. On the topic of the structure of fermion masses and mixings, string theory provides some hints towards a natural explanation. The particular case of the abelian $Z_{3}$ orbifold compactification of the heterotic superstring with two Wilson lines is especially appealing, since it predicts the standard model (SM) fermion content and correct gauge group, and offers a scenario of explicitly computable and renormalizable Yukawa couplings [1]. The latter receive exponential suppression factors that dependend on the distance between the fixed orbifold points to which the fields are attached. In this case, family replication occurs not only in the fermions, but also in the Higgs sector, and one has three families of Higgs doublets. This is a direct consequence of the orbifold compactification: since we have 27 fixed points, the twisted matter comes in 9 sets with 3 equivalent sectors in each. Having six Higgs doublets coupling to matter with distinct Yukawa couplings potentially induces dangerous flavour changing neutral currents (FCNCs) at the tree level. Therefore, the viabilty of this class of models depends not only in accommodating the current data on quark masses and mixings, but also on being in agreement with the experimental data on neutral meson mass differences (e.g. $\Delta m_{K}$ ) [2].

\section{Yukawa couplings in $Z_{3}$ orbifold models with two Wilson lines}

After Fayet-Iliopoulos (FI) breaking, the SM matter survives as the massless mode of the original Yukawa coulings. The mass matrices of the low-energy effective theory are now modified:

$$
\mathscr{M}^{u}=g N a^{u^{c}} A^{u} B^{u^{c}}, \quad \mathscr{M}^{d}=g N \varepsilon_{1} a^{d^{c}} A^{d} B^{d^{c}},
$$

where $g$ is the gauge coupling, $N$ is related to the volume of the $Z_{3}$ lattice unit cell, $\varepsilon_{i}$ are exponential suppression factors related to the shape and size of the orbifold $\left(\varepsilon_{i} \propto \exp ^{-\frac{2 \pi}{3} T_{i}}\right.$, with $T_{i}$ the lattice diagonal moduli), and $a^{f}$ are functions of the VEVs of the SM singlet scalar fields that cancel the FI $D$-term. The flavour content is encoded in

$$
A^{u} B^{u^{c}}=\left(\begin{array}{ccc}
w_{2} \varepsilon_{5} \beta^{u^{c}} & w_{6} \varepsilon_{5} & w_{4} \alpha^{u^{c}} \\
w_{6} \varepsilon_{5}^{2} \beta^{u^{c}} & w_{4} & w_{2} \alpha^{u^{c}} \\
w_{4} \varepsilon_{5}^{2} \beta^{u^{c}} & w_{2} \varepsilon_{5} & w_{6} \alpha^{u^{c}} / \varepsilon_{5}
\end{array}\right), A^{d} B^{d^{c}}=\left(\begin{array}{ccc}
w_{1} \varepsilon_{5} \beta^{d^{c}} & w_{5} \varepsilon_{5} & w_{3} \alpha^{d^{c}} \\
w_{5} \varepsilon_{5}^{2} \beta^{d^{c}} & w_{3} & w_{1} \alpha^{d^{c}} \\
w_{3} \varepsilon_{5}^{2} \beta^{d^{c}} & w_{1} \varepsilon_{5} & w_{5} \alpha^{d^{c}} / \varepsilon_{5}
\end{array}\right)
$$

In the above, $\alpha^{f}$ and $\beta^{f}$ are also functions of the singlet field VEVs, and of $\varepsilon_{5} . w_{i}$ are the VEVs of the neutral components of the six Higgs doublets. By comparing the eigenvalues of the above mass matrices (which can be analytically obtained by expanding to first order in $\varepsilon_{5}$, one can derive relations for the Higgs VEVs in terms of the down quark masses:

$$
\left\{w_{1}, w_{3}, w_{5}\right\} \propto\left\{\frac{1}{\varepsilon_{5} \beta^{d^{c}}}\left(m_{d}+\varepsilon_{5}^{5} \frac{m_{b}^{2}}{m_{s}}\right), m_{s}, \frac{m_{b} \varepsilon_{5}}{\alpha^{d^{c}}}\right\}
$$

and likewise for the up-type VEVs, in relation to the with up-quark masses. For given values of the quark masses, one fixes the ratio of the several Higgs VEVs, which in turn allows to reconstruct the 
full quark mass matrices, and obtain the quark spectra and mixings. Satisfying the current experimental bounds on the latter, imposes severe correlations on the orbifold parameters, $\left\{\varepsilon_{5}, \alpha^{f}, \beta^{f}\right\}$. Taking 4 distinct sets of input quark masses, $\left\{m_{u}, m_{d}, m_{c}, m_{s}, m_{t}, m_{b}\right\}$ (in $\mathrm{GeV}$ ) as
A : $\quad \begin{array}{llllll}0.004 & 0.008 & 1.35 & 0.13 & 180 & 4.4\end{array}$
B : $\begin{array}{llllll}0.0035 & 0.008 & 1.25 & 0.1 & 178 & 4.5\end{array}$
C : 0.00350 .0041 .150 .081764 .1
D : $\begin{array}{llllll}0.004 & 0.006 & 1.2 & 0.105 & 178 & 4.25\end{array}$

and requiring that the points are in agreement with viable quark masses and current $\left|V_{\mathrm{CKM}}\right|$ bounds [3], one obtains the following correlations on the orbifold parameters: In addition to the hierar-
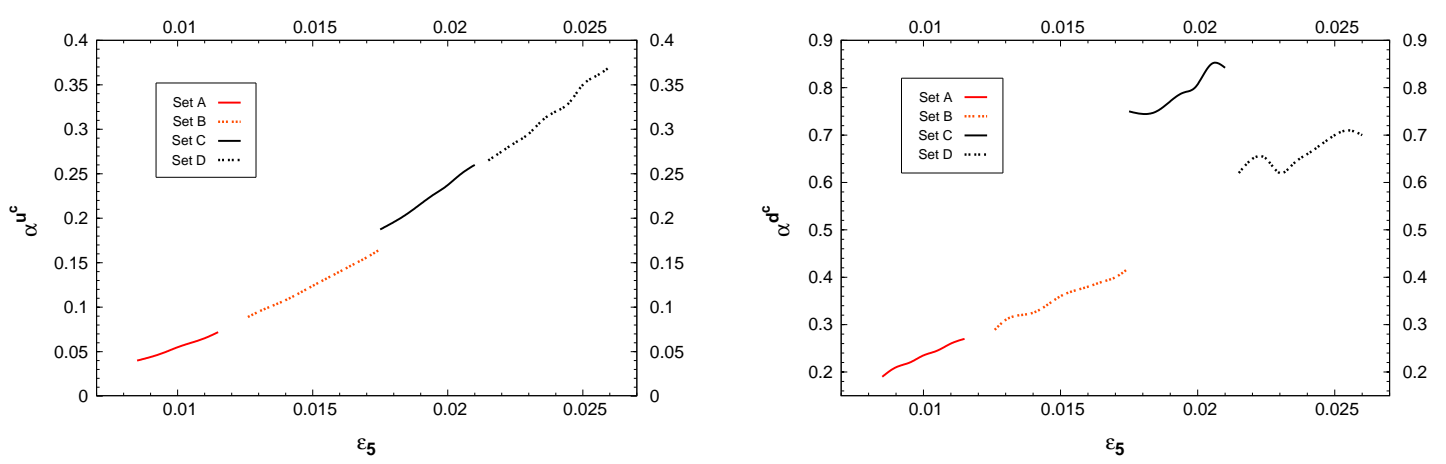

Figure 1: Correlation between the orbifold parameters $\left(\alpha^{u^{c}}, \varepsilon_{5}\right)$ and $\left(\alpha^{d^{c}}, \varepsilon_{5}\right)$ for Sets A-D.

chy constraint imposed by the observed pattern of quark masses, the VEVs must further comply with other constraints as those arising from electroweak symmetry breaking, as $\sum w_{i}^{2}=2 M_{Z}^{2} /\left(g^{2}+\right.$ $\left.g^{\prime 2}\right) \approx(174 \mathrm{GeV})^{2}$, and from defining $\tan \beta$ as $\tan \beta=\sqrt{w_{2}^{2}+w_{4}^{2}+w_{6}^{2}} / \sqrt{w_{1}^{2}+w_{3}^{2}+w_{5}^{2}}$. Bringing together the several constraints, one can derive relations that allow to extract the remaining orbifold parameters, $a^{u^{c}}, \varepsilon_{1} a^{d^{c}}$, and thus $\varepsilon_{1}$ and the moduli $T_{1,5}$. In particular, requiring that $\varepsilon_{1}$ is a perturbative parameter will disfavour regimes of large $\tan \beta$, as is clear from the Figure below.
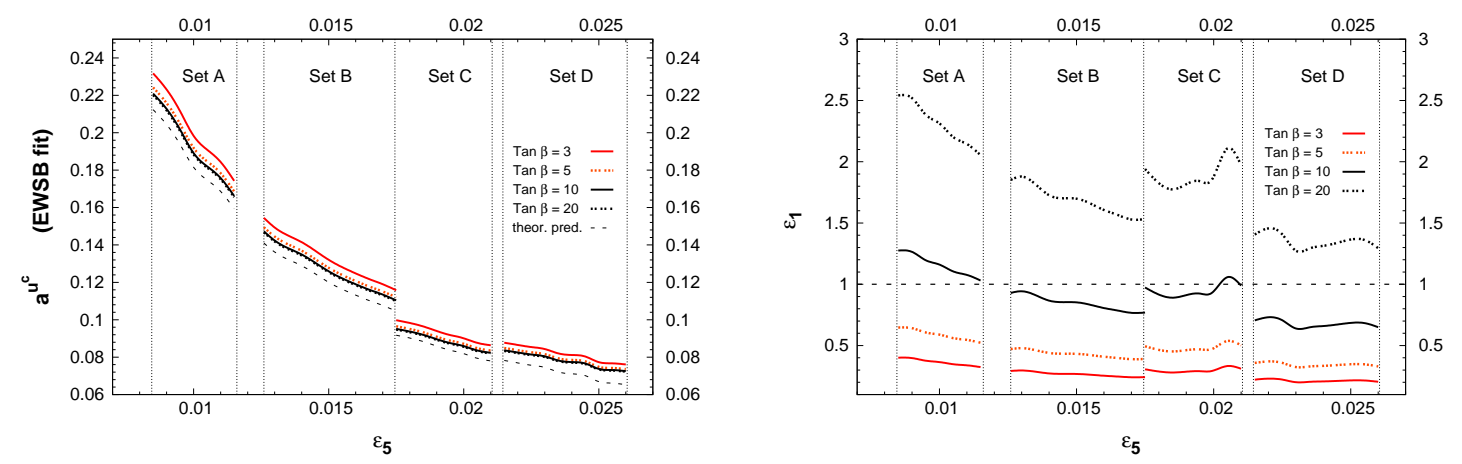

Figure 2: EW symmetry breaking predictions and theoretical estimates of $a^{u^{c}}$ (left), and EW breaking estimate of $\varepsilon_{1}$ (right) for Sets A-D and $\tan \beta=3,5,10,20$.

\section{An extended Higgs sector and FCNCs at the tree level}

A generic analysis of this class of models (which comprises 21 physical Higgs states), including minimisation of the scalar potential and derivation of the mass matrices has been conducted in 
[4]. The FCNCs induced by the tree level exchange of scalar and pseudoscalar Higgses has been evaluated, and for example, the contribution to the neutral scalar to the off diagonal element of the Kaon mass difference, $\mathscr{M}_{12}^{K}$ was found to be

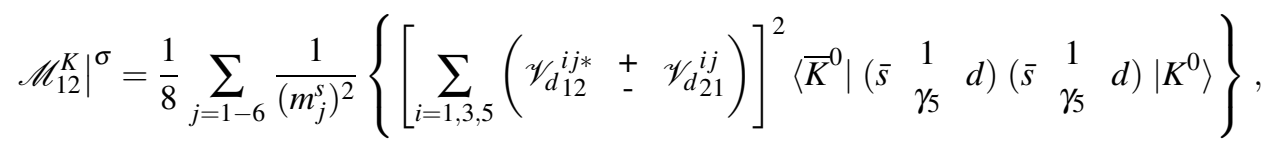

where the "+" ("-") sign is associated to the scalar (pseudoscalar) matrix element, and the flavour violation is encoded in $\mathscr{V}_{d a b}^{i j} \propto\left(V_{R}^{d} Y_{i}^{d} V_{L}^{d \dagger}\right)_{a b}$. Assuming four disting textures for the Higgs parameters, that translate in spectra whose heaviest states are (a): $760 \mathrm{GeV}$, (b): $880 \mathrm{GeV}$, (c): $1060 \mathrm{GeV}$ and (d): $800 \mathrm{GeV}$, we present in Fig.3 the models contributions to the ratios $\Delta m_{K} /\left(\Delta m_{K}\right)_{\exp }$ and $\Delta m_{B_{d}} /\left(\Delta m_{B_{d}}\right)_{\exp }$ for $\tan \beta=5$. Further analysis of other neutral meson systems, as well as that of
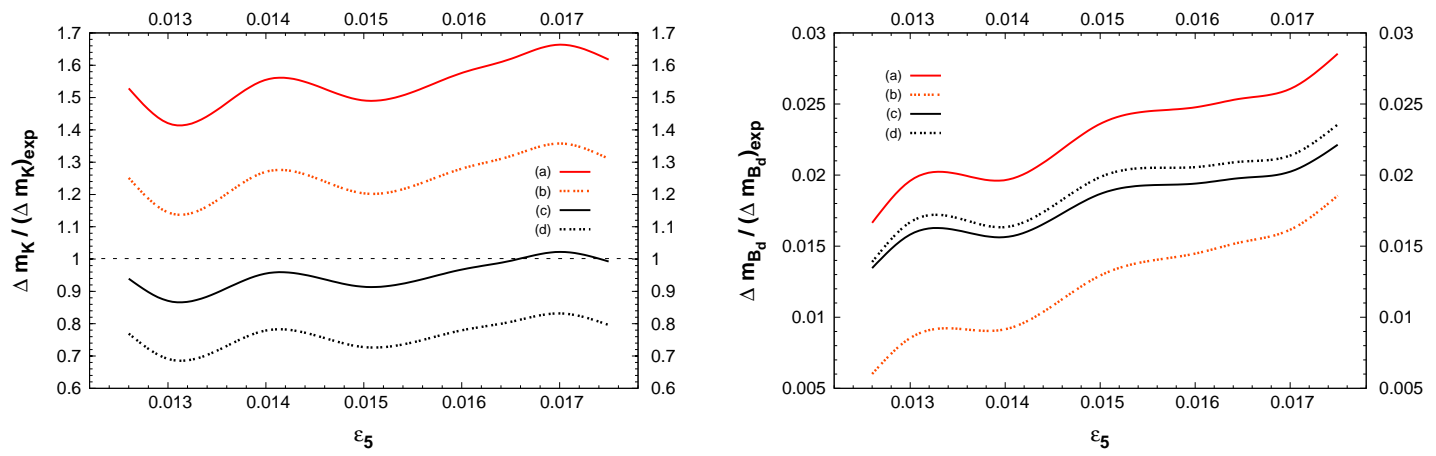

Figure 3: $\Delta m_{K} /\left(\Delta m_{K}\right)_{\text {exp }}$ and $\Delta m_{B_{d}} /\left(\Delta m_{B_{d}}\right)_{\text {exp }}$ as a function of $\varepsilon_{5}$ for Set B and Higgs textures (a)-(d).

CP violation have been also conducted in [2], finding that it is possible to have orbifold configurations in agreement with experimental data for Higgs masses not heavier than the few TeVs.

\section{Conclusions}

Orbifold models with three Higgs families are very appealing scenarios, since the observed structure of quark masses and mixings arises in a purely geometrical form from superstring constructions. We have verified that one can easily find orbifold configurations that succeed in accommodating the experimental data on quark masses and mixings, as well as neutron meson mass difference and $\mathrm{CP}$ violation without the need to call upon very Higgs textures $-m_{h}^{\max } \approx \mathscr{O}($ few TeV) - to suppress dangerous tree level FCNCs.

\section{References}

[1] S. A. Abel and C. Muñoz, "Quark and lepton masses and mixing angles from superstring constructions," JHEP 0302 (2003) 010 [arXiv:hep-ph/0212258].

[2] N. Escudero, C. Muñoz and A. M. Teixeira, "Phenomelogical viability of orbifold models with three Higgs families", FTUAM 05/18, IFT-UAM/CSIC-05-49.

[3] S. Eidelman et al. [Particle Data Group Collaboration], Phys. Lett. B 592 (2004) 1.

[4] N. Escudero, C. Muñoz and A. M. Teixeira, "FCNCs in supersymmetric multi-Higgs doublet models", FTUAM 05/17, IFT-UAM/CSIC-05-47. 